\title{
Statistical model building: Background "knowledge" based on inappropriate preselection causes misspecification
}

\author{
Lorena Hafermann ${ }^{1 *}$, Heiko Becher ${ }^{2}$, Carolin Herrmann ${ }^{1}$, Nadja Klein ${ }^{3}$, Georg Heinze ${ }^{4}$ and Geraldine Rauch ${ }^{1}$
}

\begin{abstract}
Background: Statistical model building requires selection of variables for a model depending on the model's aim. In descriptive and explanatory models, a common recommendation often met in the literature is to include all variables in the model which are assumed or known to be associated with the outcome independent of their identification with data driven selection procedures. An open question is, how reliable this assumed "background knowledge" truly is. In fact, "known" predictors might be findings from preceding studies which may also have employed inappropriate model building strategies.

Methods: We conducted a simulation study assessing the influence of treating variables as "known predictors" in model building when in fact this knowledge resulting from preceding studies might be insufficient. Within randomly generated preceding study data sets, model building with variable selection was conducted. A variable was subsequently considered as a "known" predictor if a predefined number of preceding studies identified it as relevant.

Results: Even if several preceding studies identified a variable as a "true" predictor, this classification is often false positive. Moreover, variables not identified might still be truly predictive. This especially holds true if the preceding studies employed inappropriate selection methods such as univariable selection.
\end{abstract}

Conclusions: The source of "background knowledge" should be evaluated with care. Knowledge generated on preceding studies can cause misspecification.

Keywords: Background knowledge, Univariable selection, Backward elimination, Variable selection, Regression model, Simulation study, Need for more data sharing

\section{Background}

Statistical regression models play an important role in epidemiological and medical research. The scientific aims behind those models should thereby carefully be differentiated. While explanatory models should identify causal relations and factors explaining differences in outcomes, predictive models aim at predicting an outcome variable with minimal prediction error, and descriptive models

*Correspondence: Iorena.hafermann@charite.de

${ }^{1}$ Charité - Universitätsmedizin Berlin, corporate member of Freie Universität Berlin and Humboldt-Universität zu Berlin, Institute of Biometry and Clinical Epidemiology, Charitéplatz 1, 10117 Berlin, Germany

Full list of author information is available at the end of the article ideally capture the main associations of independent variables and outcome [1]. In many applications, several aims might also be combined. In any case, consideration of the aim of model building is essential for choosing the set of independent variables for the model, as the interpretation of coefficients of the model changes with the selected companion variables $[2,3]$. In this work, we focus on the descriptive and the predictive perspective as the theory for identifying causal relations goes far beyond classical variable selection techniques [4-6].

Variable selection is an essential aspect of model building in epidemiological and medical studies. Whenever 
the number of candidate predictors seems too large for a meaningful interpretation or for a reliable prediction, the question is how to separate the truly predictive variables from the non-predictive ones and how assumed background knowledge influences this procedure. Many procedures have been proposed to automatize this step and many articles have been published addressing the performance of those procedures [2, 6-8]. As a consequence, to get an overview of the relative performance of those methods is a challenging task [9].

While general guidance on variable selection is still lacking, several articles agree on the recommendation that variable selection should always take background knowledge into account $[2,8]$. This very general recommendation must of course be adapted to specific situations, for example if the study aim is to perform confounder selection, which however will not be investigated in this work. In a systematic review screening four major epidemiological journals, Walter et al. (2009) showed that $28 \%$ of the medical studies incorporated background knowledge in their analysis [10]. Ten years later, Talbot et al. updated the review and the incorporation of prior knowledge increased to $50 \%$ [11]. The importance of prior knowledge indeed seems plausible and intuitive, especially, when there is fundamental biologic evidence for a variable being an important predictor or for being causally related to the outcome. In absence of scientifically defensible assumptions, evidence may be insufficient and based on results from few or weak preceding studies only. Walter et al. explicitly state that "Prior knowledge can be documented by referring to a study in the same population that resulted in the identification of risk factors for the outcome under study [...] or by one or more studies that identified each of the potential confounders" [10]. The level of evidence for such assumptions is, however, rarely questioned. Often, these preceding studies are also based on some kind of model building strategy producing a more or less reliable subset of identified predictors. Such assumed background knowledge, which is then transferred to the current study, is thus uncertain. An intuitive statistical approach to deal with uncertainty is the use of Bayesian methods, however in the context of modelling background knowledge these methods are seldom applied in practice. Talbot and Massamba (2019) identified only one study out of 292 included studies which incorporated background knowledge based on a Bayesian approach [11]. Therefore, it seems current practice to either include a variable as a "known" predictor or to exclude it without considering a specific prior distribution. Such an approach comes with uncertainty, which depends on the appropriateness of variable selection in the preceding studies [12]. We may therefore ask the question which model building and variable selection techniques are most often applied in preceding studies. As several systematic reviews showed [13, 14], in many studies the method of univariable selection was used meaning that predictors were identified by evaluating unadjusted associations of candidate variables with the outcome. This method is known since long to perform badly when confounding is present [15]. Another commonly used approach, which is expected to perform better, is backward elimination [15].

The objective of this paper is to evaluate the reliability of evidence on predictor selection created by preceding studies. Thereby, our interest lies in a low-dimensional setting, meaning that the number of candidate predictors is much lower than the studies' sample size. To mimic a situation often found in practice, we assumed that preceding studies identified predictors by univariable selection or backward elimination, and assessed the performance of different strategies to combine the evidence from several preceding studies by a simulation study.

\section{Methods}

We considered a data generating mechanism characterized by a linear regression model. "True" predictors are characterized by a non-zero effect representing the "true data generating mechanism". To base the below described simulation study on a realistic setting, we investigated a model resembling a real study by Sheppard et al. [16]. In there, the authors discuss that differences in blood pressure measurements occur between a measurement in a clinical environment and a measurement at home. They developed a multivariable linear regression model with the difference between diastolic blood pressure measured at home and at the clinic as the dependent variable [16]. The independent variables were age $\left(X_{\text {age }}\right)$ [years], sex $\left(X_{s e x}\right)[0 / 1]$, the first reading of the clinical blood pressure $\left(X_{c b p . f i r s t}\right)[m m h g]$, the difference of the first and a followup reading of the clinical blood pressure ( $\left.X_{\text {cbp.change }}\right)$ [mmhg], the body mass index $\left(X_{b m i}\right)\left[\frac{\mathrm{kg}}{\mathrm{m}^{2}}\right]$, the previous diagnosis of hypertension $\left(X_{\text {history }}\right)$ [0/1], the intake of antihypertensive medication $\left(X_{\text {antihyp }}\right)$ [0/1], the history of cardiovascular diseases $\left(X_{c v d}\right)$ [0/1] and the pulse pressure $\left(X_{p p}\right)[m m h g]$. We assume in the following that the data is generated by the model

$$
\begin{aligned}
Y & =36-0.08 \cdot X_{\text {age }}+3.33 \cdot X_{\text {sex }}-0.47 \cdot X_{\text {cbp.first }} \\
& +0.31 \cdot X_{\text {cbp.change }}-0.07 \cdot X_{\text {bmi }}-0.03 \cdot X_{\text {history }} \\
& +2.37 \cdot X_{\text {antihyp }}-0.4 \cdot X_{c v d}-0.06 \cdot X_{p p}+\epsilon \\
& \epsilon N\left(0, \sigma^{2}\right) .
\end{aligned}
$$

This true generating mechanism contains only the true predictors. The assumed coefficients of the above true generating mechanism were adapted from the published regression parameter estimates in the paper, but the interaction terms from the original study were excluded for the 
sake of simplicity. As a consequence, the intercept used in here deviates from the original publication in order to create reasonable values of the outcome. Moreover, the covariance structure of the exemplary model was chosen as reasonable as possible, but does not encode specific causal assumptions.

Two frequently applied variable selection methods are univariable selection and backward elimination with the Akaike Information Criterion (AIC). Although from a theoretical point of view, the Bayesian Information Criterion (BIC) as a model selection criteria may be preferred to identify the true underlying model [17], the AIC is more commonly applied in practice. In univariable selection, the final model includes only those predictors which were significant in univariable regressions. Backward elimination starts with the full model and iteratively cycles between identifying the least significant predictor and refitting the model without that predictor. The procedure is stopped if no predictor can be removed without increasing the AIC.

Note that although all variables in the true data generating mechanism are true predictors, the clinical relevance of the predictors and the size of the coefficients are different, so the impact of not selecting a true predictor is different as well. Therefore, we calculated the standardized regression coefficients of our data generating mechanism, which are 0.528 for $X_{\text {sex }},-0.406$ for $X_{c b p \text { first }}$, 0.315 for $X_{\text {antihyp }},-0.268$ for $X_{\text {age }}, 0.201$ for $X_{\text {cbp.change, }}$, -0.161 for $X_{p p},-0.093$ for $X_{b m i},-0.050$ for $X_{c v d}$ and -0.004 for $X_{\text {history. }}$. This should be kept in mind, when interpreting the simulation results below.

\section{Simulation and analysis}

Our considered simulation study consisted of three steps. In the first step, data were simulated and in steps 2 and 3 , the simulated data were analysed further. In step 1, three different "preceding" study data sets were generated according to the model specified above. This first step is highlighted in blue colour in Fig. 1. Subsequently, in step 2 , variable selection was performed within each preceding study, and for the final model of the "present" study, a variable was considered as a "known" predictor if at least one, at least two, or all three preceding studies identified it as relevant. This part of the simulation study is graphically highlighted in green in Fig. 1. In step 3, the reliability of background knowledge based on the preceding studies was evaluated with different performance indicators. Thereby, the performance indicators assess performance aspects related to descriptive and to predictive behaviour. This third step of the simulation algorithm is highlighted in orange in Fig. 1.

In the following, the three steps are described in more detail. In the first step of the simulation study, three preceding study data sets were generated according to the true data generating mechanism, including predictor and non-predictor variables as specified in the following:

\section{Step 1: data generation}

To define the candidate predictors, we additionally added a set of non-predictor variables denoted by $X_{\text {none } 1}$ to $X_{\text {none } 11}$ to the true predictor set. To simulate candidate predictors (including true predictors and non-predictor variables), we used the R-package "simdata" [18]. This package is inspired by a technical report by Binder et al. [19]. It simulates data for covariates with a predefined realistic joint distribution mimicking data from real biomedical studies. This is achieved by first drawing multivariate normal deviates with a predefinded correlation structure, and then transforming them to achieve specific realistic marginal distributions of simulated predictors and a realistic correlation structure between them. Note that the application of transformations might change the correlations. Figure 2 visualizes the respective discrete or continuous marginal densities for the simulated variables. The resulting average correlations are presented in the Supplement [Figure S1]. While the distributions of the true predictors were generated to derive clinically meaningful values in accordance with the above true generating mechanism, the distributions of non-predictor variables were chosen with the intention to create variables with complex correlation structures and a range of different distributions.

The data generating code including the applied transformations is provided in the supplemental material. For data generation, the variance $\sigma^{2}$ of the random error was set to 2 resulting in a $R^{2}$ of about 0.75 . This seemed to represent a plausible situation where still some variance is present. We considered the following specific simulation settings:

- Preceding studies with equal sample sizes

$$
n=n_{1}=n_{2}=n_{3} \text {, where } n \in\{200,500,2000\} \text {. }
$$

- In addition, we considered seven scenarios where at least two preceding studies show unequal sample sizes, where $n_{1}, n_{2}, n_{3} \in\{200,500,2000\}$.

\section{Step 2: variable selection}

Within each preceding study data set, variable selection was now performed to identify the respective predictors. We thereby relied on the following two variable selection techniques known to be often applied in applications:

- Univariable variable selection was considered with upper $p$-value thresholds of $\alpha_{i n} \in\{0.05,0.2\}$ meaning that variables which showed a $p$-value smaller or equal to $\alpha_{i n}$ were included in the full model of the preceding study.

- In addition, we also considered backward elimination with the AIC as selection criterion. 


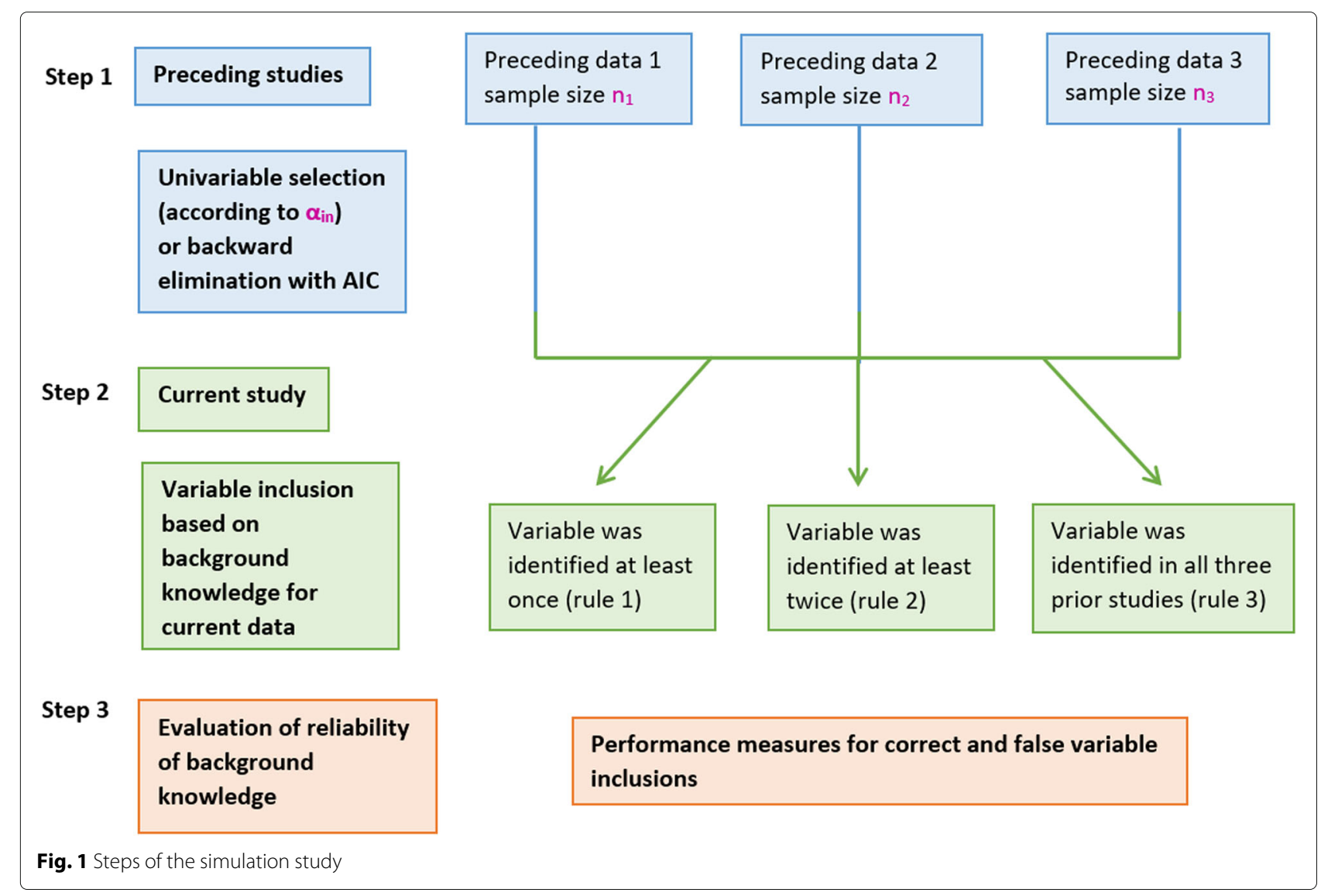

Subsequently, each of the 20 candidate predictors (9 true predictors, 11 non-predictors) was considered as a "known predictor" if it was identified by only one preceding study (rule 1), by at least two preceding studies (rule 2) or by all three preceding studies (rule 3). The set of predictors identified by these rules within the preceding studies was then considered as the set of "known predictors" (background knowledge) for the current study.

\section{Step 3: performance evaluation}

As the true predictors are known, the reliability of background knowledge based on the preceding studies was then evaluated by different performance indicators. The following different performance measures were investigated, where we focused on correct predictor identification (descriptive aim) and prediction performance (predictive aim). A discussion of suitable performance measures can also be found in [19].

- First, we evaluated how often a specific rule to quantify background knowledge from preceding studies identified all and only the true predictors, referred to as "model selection frequency" (MSF) [12]. The rates were calculated as relative frequencies over all 10,000 random replications. A value of
1 indicates that a rule is perfectly able to identify all true predictors.

- Second, we also evaluated the average relative frequency for each rule resulting in a correct identification of the true predictors referred to as "true positive rate" (TPR). Here, the identified predictor set might include additional variables with a zero effect. The rates were again calculated as relative frequencies over all random replications. The TPR is always at least as high as the MSF. A value of 1 indicates again an ideal performance.

- Third, we calculated the average false positive and false negative rates (FPR, FNR), also denoted as type I and II errors as defined in [19]. In each random replication, the number of falsely selected non-predictor variables divided by the true number of non-predictor variables (here 11) and number of falsely not-selected true predictors divided by the number of true predictor variables (here 9) were evaluated. Both numbers were then averaged over all random replications to give the FPR and FNR for a scenario, respectively. Values of 0 indicate a perfect performance.

- Fourth, we calculated the descriptive model selection frequency (DMSF), defined as the average 


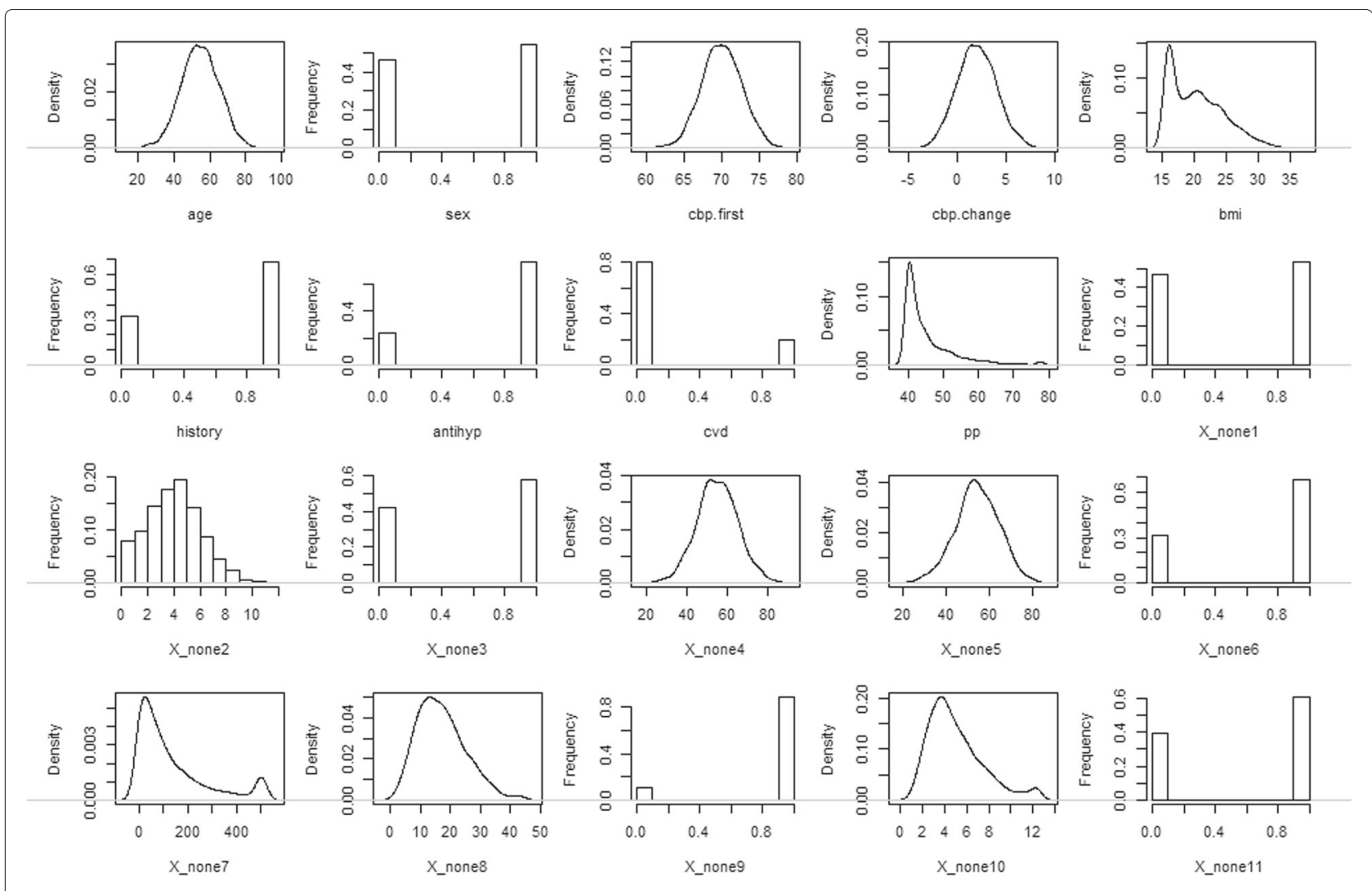

Fig. 2 Density plots of the simulated variables

relative frequency over all iterations of models, which selected the five most important predictors according to standardized regression coefficients. Considering our data generating mechanism, those five variables were $X_{\text {sex }}, X_{\text {age }}, X_{\text {cbp.first }}, X_{\text {antihyp }}$ and $X_{\text {cbp.change. }}$ Again, a value of 1 indicates perfect selection of the five most important predictors. For a model with a descriptive aim, the DMSF defines a natural performance indicator.

- Finally, we calculated the average mean square prediction error (MSPE) as the average over all simulation runs over the mean of the squared differences from the predicted and the observed outcome on a simulated data set as proposed in [19]. Therefore, we first generated a current data set of size $n=500$ for estimation of the regression coefficients which defines the proposed model. Then, we generated a validating data set and performed prediction using the proposed model. This prediction is then compared to the true outcome of the validating data set, which gives the MSPE. This procedure is repeated to define the average MSPE. A value of 0 indicates a perfect prediction. The MSPE is not bounded from above, but its value can be used to compare different models. This performance measure naturally captures the view of a predictive model, whereas for a purely descriptive model the MSPE is less important.

The investigated scenarios resulting from different simulation and analysis combinations are characterized by 1) the sample sizes of the three simulated preceding studies, 2) the variable selection technique applied for the preceding studies and 3 ) the selection criteria and threshold ( $p$-value or AIC). As we simulated three scenarios with equal sample sizes for the preceding studies and seven with unequal sample sizes, which could then all be combined with either univariable selection (considered $p$ value threshold of 0.05 or 0.2 ) or backward elimination (AIC), this resulted in a total of 30 scenarios listed in detail in Table 1. The simulations were implemented in R Version 3.5 with 10 '000 random replications for each setting and a seed of 29112018 to assure reproducibility.

\section{Results}

Table 2 shows the resulting performance measures for the 30 selected scenarios presented in Table 1.

It can be seen that independently of the scenario, the true predictor set was hardly ever selected with model selection frequencies (MSF) always lower than 0.005 
Table 1 Investigated simulation scenarios

\begin{tabular}{|c|c|c|c|c|}
\hline \multirow[t]{2}{*}{ Scenario } & \multicolumn{3}{|c|}{$\begin{array}{l}\text { Sample size in prior } \\
\text { study }\end{array}$} & \multirow[t]{2}{*}{ Variable selection used in prior study } \\
\hline & $\mathbf{n}_{1}$ & $\mathbf{n}_{2}$ & $\mathbf{n}_{3}$ & \\
\hline S1_a & 200 & 200 & 200 & Univariable selection with $p=0.05$ \\
\hline S1_b & & & & Univariable selection with $p=0.2$ \\
\hline S1_C & & & & Backward Elimination with AIC \\
\hline S2_a & 500 & 500 & 500 & Univariable selection with $p=0.05$ \\
\hline S2_b & & & & Univariable selection with $p=0.2$ \\
\hline $\mathrm{S} 2{ }_{-} \mathrm{C}$ & & & & Backward Elimination with AIC \\
\hline S3_a & 2000 & 2000 & 2000 & Univariable selection with $p=0.05$ \\
\hline S3_b & & & & Univariable selection with $p=0.2$ \\
\hline S3_C & & & & Backward Elimination with AIC \\
\hline S4_a & 200 & 200 & 500 & Univariable selection with $p=0.05$ \\
\hline S4_b & & & & Univariable selection with $p=0.2$ \\
\hline S4_C & & & & Backward Elimination with AIC \\
\hline S5_a & 200 & 500 & 500 & Univariable selection with $p=0.05$ \\
\hline S5_b & & & & Univariable selection with $p=0.2$ \\
\hline S5_C & & & & Backward Elimination with AIC \\
\hline S6_a & 200 & 500 & 2000 & Univariable selection with $p=0.05$ \\
\hline S6_b & & & & Univariable selection with $p=0.2$ \\
\hline S6_c & & & & Backward Elimination with AIC \\
\hline S7_a & 200 & 200 & 2000 & Univariable selection with $p=0.05$ \\
\hline S7_b & & & & Univariable selection with $p=0.2$ \\
\hline S7_C & & & & Backward Elimination with AIC \\
\hline S8_a & 500 & 500 & 2000 & Univariable selection with $p=0.05$ \\
\hline S8_b & & & & Univariable selection with $p=0.2$ \\
\hline S8_c & & & & Backward Elimination with AIC \\
\hline S9_a & 200 & 2000 & 2000 & Univariable selection with $p=0.05$ \\
\hline S9_b & & & & Univariable selection with $p=0.2$ \\
\hline S9_c & & & & Backward Elimination with AIC \\
\hline S10_a & 500 & 2000 & 2000 & Univariable selection with $p=0.05$ \\
\hline S10_b & & & & Univariable selection with $p=0.2$ \\
\hline S10_c & & & & Backward Elimination with AIC \\
\hline
\end{tabular}

for univariable selection and values lower than 0.04 for backward elimination (columns 2, 6, 10).

Models containing all but not only true predictors were identified more often as indicated by TPR values well above MSF (columns 3, 7, 11). In the comparison of the different rules, rule 1 was clearly the best with respect to the TPR across all scenarios followed by rule 2 and rule 3 . This is intuitive as a model that only contains predictors identified by at least one preceding study (rule 1) is more likely to contain all true predictors than if the selection is more restrictive. Across all scenarios, the univariable selection with $\alpha_{i n}=0.2$ showed the highest TPR with ranges of 0.644 to 0.992 for the most favourable rule 1 , whereas univariable selection with $\alpha_{\text {in }}=0.05$ led to TPR in the range between 0.247 to 0.912 and backward elimination showed the worst TPR with a range of 0.325 to 0.475 for rule 1 .

In contrast, rule 1 turned out to be the worst in terms of FPR. Moreover, a higher $p$-value threshold for univariable selection resulted in increased average FPR (columns 4, 


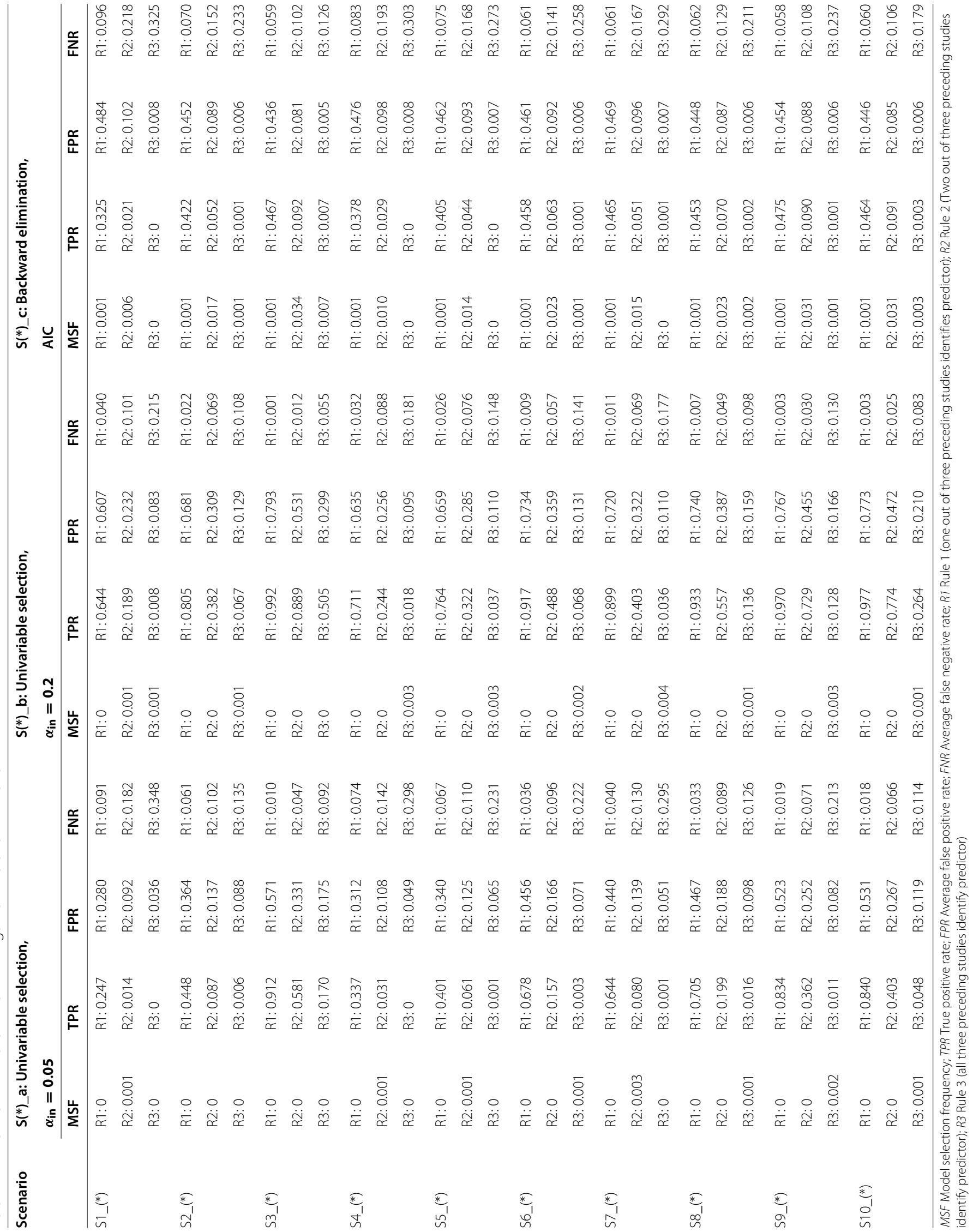


8). Backward elimination showed average FPR that were a little lower than those for univariable selection with $\alpha_{i n}=$ 0.2 (columns 8, 12).

At the same time, a higher $p$-value threshold for univariable selection naturally resulted in lower FNR (columns 5 , 9). For backward elimination, higher average FNR were observed than for univariable selection with $\alpha_{i n}=0.2$ (columns 9, 13). Since for predictive models a low FNR is desirable, rule 1 and a higher $p$-value threshold for univariable selection would be preferred.

Whereas Table 2 provides performance measures across the complete set of candidate predictors, in Fig. 3 the rates of inclusion for the individual variables (true predictors and non-predictors) are graphically summarized as functions of the preceding study sample size (with assumed equal sample sizes in all three preceding studies).

Figure 3 shows how well the different selection methods and rules could yield a separation between the true predictors and the non-predictors. Ideally, the coloured lines (true predictors) should take values close to 1 whereas all black lines should be close to 0 . Generally, it can be observed that backward elimination allowed for the best differentiation between true predictors and nonpredictors, whereas the univariable selection approaches behaved similar, but a better separation was achieved with $\alpha_{\text {in }}=0.05$.

The univariable selection approaches tended to overlook the true predictor $X_{\text {cbp.change }}$, with corresponding true regression coefficient of 0.31 , whereas backward elimination did discover it. In contrast, for backward elimination $X_{\text {history }}$ with a regression coefficient of $-0.03 \mathrm{had}$ the smallest inclusion frequency. Univariable selection, however, did identify it correctly. This can be explained as $X_{\text {history }}$ was highly correlated to $X_{\text {sex }}$, which had a high inclusion frequency. There is the general tendency that the more preceding studies were required to identify the same predictor, the better the differentiation between true predictors and non-predictors became. In this view, rule 3 was the best, followed by rule 2 and rule 1 .

For a model with a descriptive aim, it is important to capture the variables that exhibit the strongest associations with the outcome in the multivariable context. This was evaluated with the DMSF reported in Table 3. Here, we identified a clear advantage of backward elimination.

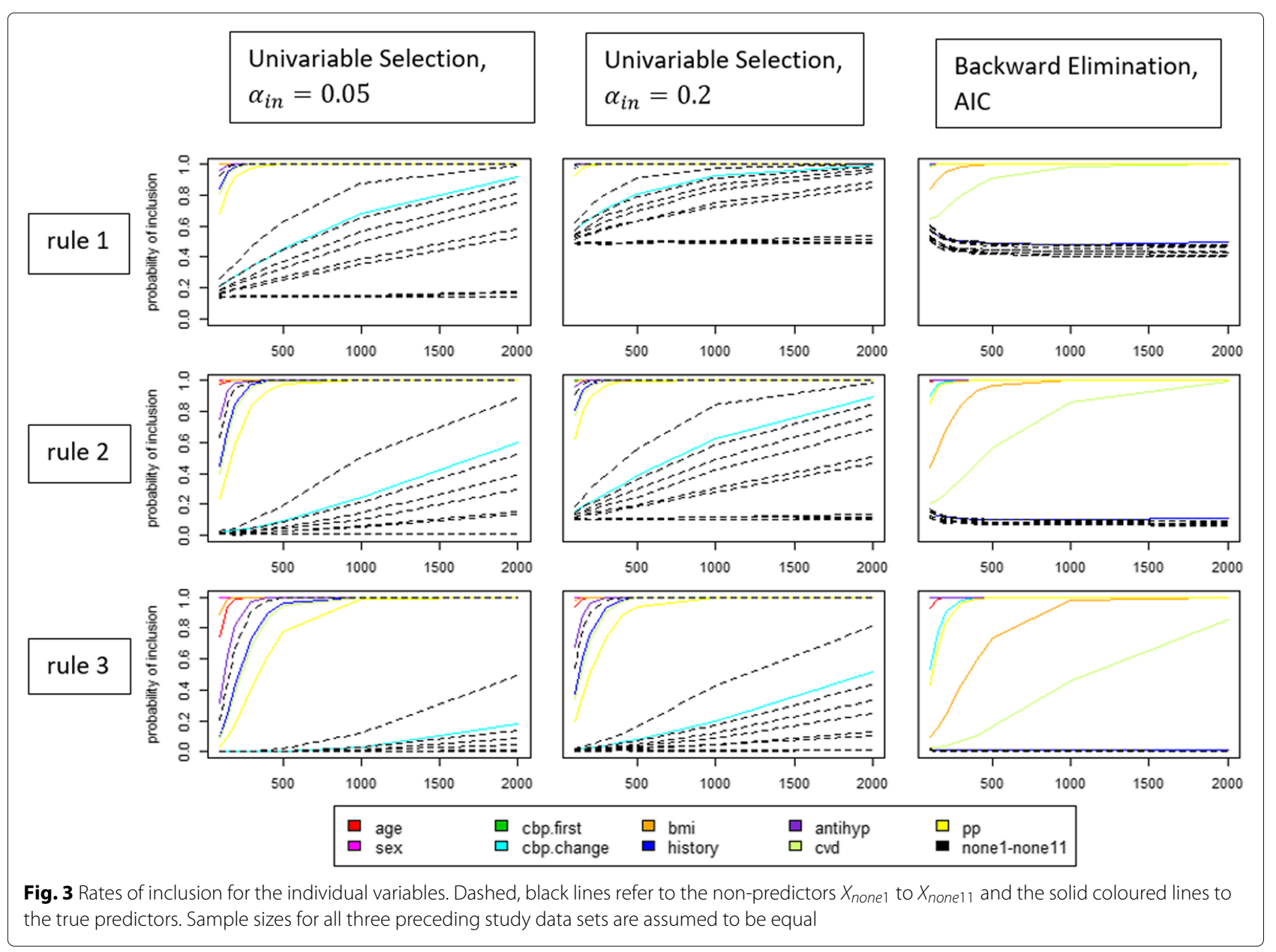


Table 3 Descriptive model selection frequencies (DMSF) for investigated simulation scenarios

\begin{tabular}{|c|c|c|c|}
\hline Scenario & $\begin{array}{l}S\left({ }^{*}\right) \_a: \text { Univariable selection, } \\
\alpha_{\text {in }}=0.05\end{array}$ & $\begin{array}{l}\mathrm{S}\left({ }^{*}\right) \_b: \text { Univariable selection, } \\
\alpha_{\text {in }}=0.2\end{array}$ & $\begin{array}{l}S\left({ }^{*}\right) \text { C: Backward elimination, } \\
\text { AIC }\end{array}$ \\
\hline \multirow[t]{3}{*}{ S1_(*) } & $\mathrm{R} 1: 0.271$ & R1: 0.648 & $\mathrm{R} 1: 1$ \\
\hline & R2: 0.028 & $R 2: 0.212$ & R2: 0.997 \\
\hline & R3: 0.001 & R3: 0.024 & R3: 0.894 \\
\hline \multirow[t]{3}{*}{ S2_(*) } & R1: 0.448 & R1: 0.805 & R1: 1 \\
\hline & R2: 0.086 & R2: 0.382 & $\mathrm{R} 2: 1$ \\
\hline & R3: 0.006 & R3: 0.069 & R3: 0.999 \\
\hline \multirow[t]{3}{*}{ S3_(*) } & R1: 0.912 & R1: 0.992 & R1: 1 \\
\hline & R2: 0.581 & R2: 0.889 & R2: 1 \\
\hline & R3: 0.170 & R3: 0.505 & R3: 1 \\
\hline \multirow[t]{3}{*}{ S4_(*) } & R1: 0.340 & $\mathrm{R} 1: 0.711$ & $\mathrm{R} 1: 1$ \\
\hline & R2: 0.044 & R2: 0.255 & R2: 0.999 \\
\hline & R3: 0.001 & R3: 0.037 & R3: 0.929 \\
\hline \multirow[t]{3}{*}{ S5_(*) } & R1: 0.401 & $\mathrm{R} 1: 0.764$ & R1: 1 \\
\hline & R2: 0.064 & R2: 0.323 & R2: 1 \\
\hline & R3: 0.003 & R3: 0.054 & R3: 0.966 \\
\hline \multirow[t]{3}{*}{ S6_(*) } & R1: 0.678 & R1: 0.917 & $\mathrm{R} 1: 1$ \\
\hline & R2: 0.161 & R2: 0.489 & $\mathrm{R} 2: 1$ \\
\hline & R3: 0.009 & R3: 0.097 & R3: 0.959 \\
\hline \multirow[t]{3}{*}{ S7_(*) } & R1: 0.644 & R1: 0.899 & R1: 1 \\
\hline & R2: 0.107 & R2: 0.419 & R2: 0.998 \\
\hline & R3: 0.005 & R3: 0.072 & R3: 0.929 \\
\hline \multirow[t]{3}{*}{ S8_(*) } & R1: 0.705 & R1: 0.933 & R1: 1 \\
\hline & R2: 0.200 & R2: 0.557 & R2: 1 \\
\hline & R3: 0.019 & R3: 0.140 & R3: 1 \\
\hline \multirow[t]{3}{*}{ S9_(*) } & R1: 0.834 & R1: 0.970 & R1: 1 \\
\hline & R2: 0.362 & R2: 0.729 & $\mathrm{R} 2: 1$ \\
\hline & R3: 0.029 & R3: 0.184 & R3: 0.965 \\
\hline \multirow[t]{3}{*}{ S10_(*) } & R1: 0.840 & R1: 0.977 & R1: 1 \\
\hline & R2: 0.403 & R2: 0.774 & R2: 1 \\
\hline & R3: 0.052 & R3: 0.267 & R3: 1 \\
\hline
\end{tabular}

R1 Rule 1 (one out of three preceding studies identifies predictor); R2 Rule 2 (Two out of three preceding studies identify predictor); R3 Rule 3 (all three preceding studies identify predictor)

Across all scenarios using backward elimination the DMSF ranged from 0.895 to 1 whereas for univariable selection with an $\alpha_{i n}=0.05$ the DMSF decreased to 0.001 for rule 3 .

In order to evaluate the predictive performance of the models, we report the MSPE in Table 4. The only clear result is that the MSPE is always the lowest for rule 1 followed by rule 2 and rule 3 in all scenarios. This is in line with expectations as rule 1 naturally selects models with larger numbers of predictors than rules 2 and 3 . The MSPE does not generally decrease with sample size of the preceding studies, which is due to the fact that for the current data set a fixed sample size of 500 was used. Moreover, there is no clear advantage of any selection procedure used in the preceding studies.

Still, for reasonable preceding study sample sizes of 500 , the probabilities of inclusion for the true predictors were often considerably below 1 for all rules and selection 
Table 4 Mean square prediction error for investigated simulation scenarios

\begin{tabular}{|c|c|c|c|}
\hline Scenario & $\begin{array}{l}S\left({ }^{*}\right) \_a: \text { Univariable selection, } \\
\alpha_{\text {in }}=0.05\end{array}$ & $\begin{array}{l}S\left({ }^{*}\right) \_b: \text { Univariable selection, } \\
\alpha_{\text {in }}=0.2\end{array}$ & $\begin{array}{l}S\left({ }^{*}\right) \_c: \text { Backward elimination, } \\
\text { AIC }\end{array}$ \\
\hline \multirow[t]{3}{*}{ S1_(*) } & $\mathrm{R} 1: 4.521$ & $\mathrm{R} 1: 4.173$ & R1: 4.293 \\
\hline & R2: 4.669 & R2: 4.362 & $\mathrm{R} 2: 4.250$ \\
\hline & R3: 4.933 & R3: 4.536 & R3: 4.307 \\
\hline \multirow[t]{3}{*}{ S2_(*) } & R1: 4.252 & R1: 4.151 & R1: 4.227 \\
\hline & R2: 4.349 & R2: 4.147 & R2: 4.186 \\
\hline & R3: 4.401 & R3: 4.183 & R3: 4.231 \\
\hline \multirow[t]{3}{*}{ S3_(*) } & R1: 4.088 & $\mathrm{R} 1: 4.466$ & $\mathrm{R} 1: 4.278$ \\
\hline & R2: 4.157 & $R 2: 4.503$ & R2: 4.244 \\
\hline & R3: 4.265 & R3: 4.683 & R3: 4.239 \\
\hline \multirow[t]{3}{*}{ S4_(*) } & R1: 4.102 & R1: 4.133 & R1: 3.942 \\
\hline & R2: 4.261 & R2: 4.320 & R2: 3.966 \\
\hline & R3: 4.604 & R3: 4.485 & R3: 4.038 \\
\hline \multirow[t]{3}{*}{ S5_(*) } & R1: 4.319 & R1: 4.144 & R1: 4.231 \\
\hline & R2: 4.409 & $R 2: 4.258$ & R2: 4.198 \\
\hline & R3: 4.686 & R3: 4.419 & R3: 4.297 \\
\hline \multirow[t]{3}{*}{ S6_(*) } & R1: 4.460 & R1: 4.133 & R1: 4.000 \\
\hline & R2: 4.479 & R2: 4.162 & R2: 4.335 \\
\hline & R3: 4.727 & R3: 4.303 & R3: 4.377 \\
\hline \multirow[t]{3}{*}{ S7_(*) } & R1: 4.342 & R1: 4.111 & R1: 4.223 \\
\hline & R2: 4.665 & $R 2: 4.215$ & R2: 4.234 \\
\hline & R3: 4.993 & R3: 4.429 & R3: 4.325 \\
\hline \multirow[t]{3}{*}{ S8_(*) } & R1: 4.127 & $\mathrm{R} 1: 4.864$ & R1: 4.083 \\
\hline & R2: 4.251 & R2: 4.965 & R2: 4.089 \\
\hline & R3: 4.429 & R3: 5.119 & R3: 4.113 \\
\hline \multirow[t]{3}{*}{ S9_(*) } & $\mathrm{R} 1: 4.074$ & $\mathrm{R} 1: 4.619$ & R1: 4.012 \\
\hline & R2: 4.167 & R2: 4.617 & R2: 3.998 \\
\hline & R3: 4.369 & R3: 4.767 & R3: 4.061 \\
\hline \multirow[t]{3}{*}{ S10_(*) } & R1: 4.058 & R1: 4.047 & R1: 4.018 \\
\hline & R2: 4.142 & R2: 4.115 & R2: 3.991 \\
\hline & R3: 4.212 & R3: 4.337 & R3: 4.009 \\
\hline
\end{tabular}

R1 Rule 1 (one out of three preceding studies identifies predictor); R2 Rule 2 (Two out of three preceding studies identify predictor); R3 Rule 3 (all three preceding studies identify predictor)

techniques. In addition, the probabilities of inclusion for the non-predictors were mostly considerably above 0 for all rules combined with univariable selection.

\section{Discussion}

The results of our simulation study suggest that the variable selection techniques used in preceding studies may have major effects on the level of evidence for what is called background knowledge. In our study, we investigated how inappropriate selection methods in such preceding studies can translate into poor representation of background knowledge in a given study. The simulation study showed that when the commonly applied univariable selection was used, the identified set of variables might not be reliable, even when several preceding studies have identified the same predictor. The stability of the identified predictor set is also limited if a more appropriate selection method such as backward elimination 
has been applied. Our results showed that choosing only variables which have been pre-identified multiple times does not necessarily improve the rates of correct inclusion of true predictors in general, but only reduces the rate of wrong inclusion for non-predictors. Moreover, our results show that the predictive performance of the resulting models is also limited independent of the variable selection procedure in the preceding studies.

The identification of true predictors by one or several preceding studies also depends on the underlying sample size and the number of candidate predictors. In the literature, the ratio of sample size and candidate variables is assessed via the ratio of "events per variable" (EPV). A rule of thumb says that at least 10 observations are required per variable in linear regression models [20]. This implies that for the situation considered in here with 20 candidate variables, the sample size should be at least 200. However, with preceding studies of that size, results were not yet satisfactory in our simulation study. Sometimes even a recommendation of at least $50 \mathrm{EPV}$ is given, which is better in line with our results [21]. Recent development even goes one step further, Riley et al. (2020) state that the calculation of the sample size should also incorporate other factors like the expected predictive performance of the model [22]. Here, results improved only if very high sample sizes of 1000 or more were assumed, which in some practical situations may not be achievable.

Thus, results shown here are limited for various reasons. A very general point of criticism regarding simulation studies is the assumption of the existence of a true underlying model. Several authors already declared that they do not agree with this assumption [2, 21, 23]. Nonetheless, when evaluating the performance of a model, the assumption of different data generating mechanisms helps in understanding and comparing properties of the evaluated model building strategies. Despite analysing a broad variety of scenarios, simulation studies are never able to cover all possible settings eventually found in applications. In here, the same simulation design was applied for all three preceding data sets, whereas changes in the correlation structure and the variable distributions might have an impact.

Further, the relative amount of non-predictors impacts the performance. We also investigated different settings with fewer non-predictors referring to a lower signal-tonoise ratio (results not shown). Even in this setting, where it should be easier to identify the true predictors, the performance measures indicated no relevant improvement compared to the scenarios shown here.

In this work, we concentrated on a multivariable linear regression model. For future research, other regression model types with a nonlinear link function (logistic, Cox regression) implying noncollapsibility issues could be investigated. We assume that with such models, selection uncertainty is even higher.

We have focused on background knowledge from a black-or-white perspective, that is a predictor is either included or not. Incorporating the uncertainty of background knowledge could most naturally be done by using Bayesian hierarchical models, as e.g. done by Gracia et al. (2015) [24] or using informative prior distributions in an empirical Bayes approach [25]. Unfortunately, those methods are rarely used in clinical research. As the aim of our work was to assess the impact of commonly applied methods, Bayesian methods were not further investigated although they are worthwhile to be considered in applications.

As a further limitation, we only considered low dimensional data. Generally, methodological results on model building for low and high dimensional data might deviate [26] so that our results cannot be transferred easily to the situation of high dimensional data. A similar investigation of background knowledge in variable selection for high-dimensional omics data was conducted by Liu et al. [27]. They proposed to integrate background knowledge along with marginal correlations in the prescreening procedure of omics data before applying the LASSO. A similar method was proposed by Bergersen et al. [28] who directly integrated background knowledge by weighting the penalties of each regression coefficient.

Note that within this work, we were interested in identifying the correct set of predictors and/or a good prediction which is important in the context of descriptive and predictive models. We did not focus on explanatory models and therefore did not assess the accuracy in estimation of the regression coefficients. However, one interesting aspect would be to look at the common recommendation to control for all variables that are causes of either the exposure or the outcome and not on the causal pathway [29]. Nevertheless, poor methodology in preceding studies may also increase the risk to not correctly identify and specify confounders in an explanatory study.

\section{Conclusion}

To conclude, we strongly advised to identify the source and the level of evidence for so called "background knowledge". If background knowledge is only based on a few preceding studies without sufficient biological support, the methodology of these studies should be carefully investigated, and uncertainties related to the selection or non-selection of variables in such studies should be critically inferred [12]. This does not imply a recommendation against the incorporation of background knowledge in model building, but rather aims at making researchers more sensitive to a critical appraisal of the existing evidence. 


\section{Supplementary Information}

The online version contains supplementary material available at

https://doi.org/10.1186/s12874-021-01373-z.

Additional file 1: S 1: Average correlation coefficients for the resulting transformed candidate predictors. S 2: Data simulating R code.

\section{Acknowledgements}

Not applicable.

\section{Authors' contributions}

LH implemented the conception and design of the study, and conducted the analysis and interpretation of data, drafted the article and finally approved it. $\mathrm{HB}, \mathrm{CH}, \mathrm{GH}$ and NK supported the conception of the study and the analysis and interpretation of data, they critically revised the manuscript draft and approved the final version. GR provided the ideas and supervised the conception and design of the study, the analysis and interpretation of data, drafted the article and finally approved it. The authors read and approved the final manuscript.

\section{Funding}

This work was supported through the joint German-Austrian DFG and FWF project [DFG: RA-2347/8-1] to Geraldine Rauch, [FWF: I-4739-B] to Georg Heinze and [DFG: BE-2056/22-1] to Heiko Becher. Apart from this project, this research received no additional funding. Open Access funding enabled and organized by Projekt DEAL.

\section{Availability of data and materials}

No original trial data are used in this work. Simulated data and software source code that support the findings of the simulation study can be found in the supplemental material.

\section{Declarations}

Ethics approval and consent to participate

Not applicable.

\section{Consent for publication}

Not applicable.

\section{Competing interests}

The authors declare that they have no competing interests.

\section{Author details}

${ }^{1}$ Charité - Universitätsmedizin Berlin, corporate member of Freie Universität Berlin and Humboldt-Universität zu Berlin, Institute of Biometry and Clinical Epidemiology, Charitéplatz 1, 10117 Berlin, Germany. ${ }^{2}$ Institute of Medical Biometry and Epidemiology, University Hospital Hamburg-Eppendorf, Martinistraße 52, 20246 Hamburg, Germany. ${ }^{3}$ Humboldt Universität zu Berlin School of Business and Economics, Unter den Linden 6, 10099 Berlin, Germany. ${ }^{4}$ Section for Clinical Biometrics, Center for Medical Statistics, Informatics and Intelligent Systems, Medical University of Vienna, Spitalgasse 23, 1090 Vienna, Austria.

Received: 19 November 2020 Accepted: 17 August 2021

Published online: 29 September 2021

\section{References}

1. Shmueli G. To Explain or to Predict? Stat Sci. 2010;25(3):289-310

2. Heinze $G$, Wallisch C, Dunkler D. Variable selection - A review and recommendations for the practicing statistician. Biom J. 2018;60(3): 631-69.

3. le Cessie S, Luijken K, Goetghebeur E. Regarding "Variable selection - A review and recommendations for the practicing statistician" by G. Heinze, C. Wallisch, and D. Dunkler. Biom J. 2019;61(6):1595-97.

4. Greenland S. Modeling and variable selection in epidemiologic analysis. Am J Public Health. 1989;79(3):340-49.

5. Shrier I, Platt R. Reducing bias through directed acyclic graphs. BMC Med Res Methodol. 2008;8(70):1-15.
6. Witte J, Didelez V. Covariate selection strategies for causal inference: Classification and comparison. Biom J. 2019;61(5):1270-89.

7. Mantel N. Why Stepdown Procedures in Variable Selection. Technometrics. 1970;12(3):621-25.

8. Royston P, Sauerbrei W. Multivariable model-building: A pragmatic approach to regression analysis based on fractional polynomials for continuous variables. Chichester: John Wiley \& Sons: Repr. Wiley Series in Probability and Statistics; 2009.

9. Sauerbrei W, Perperoglou A, Schmid M, Abrahamowicz M, Becher $\mathrm{H}_{\text {, }}$ Binder $\mathrm{H}$, et al. State of the art in selection of variables and functional forms in multivariable analysis - outstanding issues. Diagn Progn Res. 2020:4(1):1-18.

10. Walter $\mathrm{S}$, Tiemeier $\mathrm{H}$. Variable selection: current practice in epidemiological studies. Eur J Epidemiol. 2009:24(12):733-36.

11. Talbot D, Kubuta Massamba V. A descriptive review of variable selection methods in four epidemiologic journals: there is still room for improvement. Eur J Epidemiol. 2019;34(8):725-30.

12. Wallisch C, Dunkler D, Rauch G, de Bin R, Heinze G. Selection of variables for multivariable models: Opportunities and limitations in quantifying model stability by resampling. Stat Med. 2021;40(2):369-81.

13. Bouwmeester W, Zuithoff N, Mallett S, Geerlings MI, Vergouwe $Y$, Steyerberg EW, et al. Reporting and methods in clinical prediction research: a systematic review. PLoS Med. 2012;9(5):1-12.

14. Mallett S, Royston P, Dutton S, Waters R, Altman DG. Reporting methods in studies developing prognostic models in cancer: a review. BMC Medicine. 2010;8(20):1-11.

15. Sun G, Shook TL, Kay GL. Inappropriate use of bivariable analysis to screen risk factors for use in multivariable analysis. J Clin Epidemiol. 1996:69(8):907-16.

16. Sheppard JP, Stevens R, Gill P, Martin U, Godwin M, Hanely J, et al Predicting Out-of-Office Blood Pressure in the Clinic (PROOFBP): Derivation and Validation of a Tool to Improve the Accuracy of Blood Pressure Measurement in Clinical Practice. Hypertension. 2016;67(5): 961-50.

17. Vrieze SI. Model selection and psychological theory: A discussion of the differences between the Akaike Information Criterion (AIC) and the Bayesian Information Criterion (BIC). Psychol Methods. 2012;17(2):228-43.

18. Kammer M. R-package simdata. 2020. https://github.com/matherealize/ simdata. Accessed 8 Apr 2021

19. Binder H, Sauerbrei W, Royston P. Multivariable model-building with continuous covariates: 1. Performance measures and simulation design: Technical Report FDM-Preprint 105: University of Freiburg Germany; 2011. Retrieved from http://www.fdm.uni-freiburg.de/publications-preprints/ papers/pre105.

20. Austin PC, Steyerberg EW. The number of subjects per variable required in linear regression analyses. J Clin Epidemiol. 2015;68(6):627-36.

21. Steyerberg E. Clinical prediction models: A practical approach to development, validation, and updating. New York: Springer; 2009.

22. Riley RD, Ensor J, Snell KIE, Harrell FE, Martin GP, Reitsma JB, et al. Calculating the sample size required for developing a clinical prediction model. BMJ. 2020:368:1-12.

23. Burnham KP, Anderson DR. Model selection and multimodel inference: $A$ practical information- theoretic approach. New York: Springer; 2002.

24. Gracia E, López-Quílez A, Marco M, Lladosa S, Lila M. The spatial epidemiology of intimate partner violence: do neighborhoods matter? Am J Epidemiol. 2015;182(1):58-66.

25. Hill SM, Neve RM, Bayani N, Kuo W, Ziyad S, Spellman PT, et al. Integrating biological knowledge into variable selection: an empirical Bayes approach with an application in cancer biology. BMC Bioinformatics. 2012;13(94):1-16.

26. Sauerbrei W, Boulesteix A, Binder H. Stability investigations of multivariable regression models derived from low- and high-dimensional data. J Biopharm Stat. 2011:21(6):1206-31.

27. Liu C, Jiang J, Gu J, de Yu Z, Wang T, Lu H. High-dimensional omics data analysis using a variable screening protocol with prior knowledge integration (SKI). BMC Syst Biol. 2016;10(118):457-64.

28. Bergersen LC, Glad IK, Lyng H. Weighted lasso with data integration. Stat Appl Genet Mol Biol. 2011;10(1):1-29.

29. VanderWeele TJ, Shpitser I. A new criterion for confounder selection. Biometrics. 2011;67(4):1406-13.

\section{Publisher's Note}

Springer Nature remains neutral with regard to jurisdictional claims in published maps and institutional affiliations. 